SHORT REPORT

\title{
Adult onset Niemann-Pick disease type $C$ presenting with psychosis
}

\author{
K A Josephs, M W Van Gerpen, J A Van Gerpen
}

J Neurol Neurosurg Psychiatry 2003;74:528-529

Niemann-Pick disease type C (NPC) is an autosomal recessive neurometabolic disorder that rarely presents in adulthood, and is associated with cognitive decline, various movement disorders (ataxia, chorea, dystonia, and myoclonus), a vertical supranuclear gaze palsy (VSGP), and seizures. A recent case report demonstrated a delay in diagnosis of eight years when a patient with NPC presented with psychosis. This article reviewed all cases seen at the Mayo Clinic with a possible diagnosis of NPC between 1976 and 2000. Of the 52 possible cases, five had an established diagnosis of adult onset NPC. Of these, two presented with psychosis and were not diagnosed with NPC for 5 and 15 years, respectively. NPC may initially present in adulthood with psychosis, and when psychosis is associated with VSGP, various dyskinesias, and seizures, NPC should be suspected.

$\mathrm{N}$ iemann Pick disease Type C (NPC) is an autosomal recessive neurometabolic disorder associated with cognitive decline and movement disorders. ${ }^{1}$ Most adult NPC patients evaluated in movement disorder clinics developed their initial symptoms in childhood and have had a slowly progressive course. Adult onset NPC patients may be difficult to diagnose, particularly if they present with psychosis, have been exposed to neuroleptics and subsequently develop dyskinesias. Shulman and collegues, ${ }^{2}$ described such a patient, who carried a diagnosis of schizophrenia for eight years before the correct diagnosis of NPC was made. We present two additional cases of adult onset, cytochemically confirmed NPC, in which psychosis was the initial symptom. As both patients had been exposed to neuroleptics, their subsequent movement disorders were attributed to a tardive phenomenon. The diagnosis of NPC was delayed for 15 years in the first case and five years in the second.

We reviewed the Mayo Clinic experience with adult onset NPC patients presenting with psychosis.

\section{METHOD}

The Mayo Clinic electronic record system was used to identify all patients with a possible diagnosis of NPC seen at Mayo Clinic Rochester between 1976 and 2000. Possible NPC was defined as any paediatric or adult case in which a diagnosis of NPC was felt probable given the clinical presentation and either bone marrow biopsy demonstrating sea blue histiocytes/foamy macrophages or diagnosis was established by cholesterol esterification technique, filipin staining, mutational analysis of the NPC gene, or confirmed by necopsy. Fifty two such patients were identified and their charts reviewed. Of these 52 cases, five cases were of adult onset (defined as the occurrence of the first symptom/s at, or after, age 18 years) and had an established diagnosis of NPC as defined above.

Psychosis was defined as the presence of delusions, hallucinations, illusions, or paranoia. Psychosis must have been one of the presenting symptoms.

\section{RESULTS}

Of these five adult onset NPC cases two presented with psychosis.

\section{Case 1}

A 61 year old woman was transferred to the Mayo Clinic with a 15 year history of an undiagnosed, progressive neuropsychiatric disorder, rendering her bed bound and unable to eat.

The patient initially developed depression and hypersomnolence at age 46. Her symptoms improved with an antidepressant. At 49, she was hospitalised for mood lability, loquacity, delusions, and hypervigilance. She was treated with thiothixine, imipramine, and lithium then discharged. At age 50, she developed a gait disorder with postural instability, was noted to "lean backwards" and have retropulsion. This was attributed to tardive parkinsonism. She was treated with levodopa and bromocriptine, and her gait improved. However, she again became hypersomnolent, as well as dysphagic, which required gastrostomy tube placement. One year later, auditory hallucinations began, and she became paranoid, hyperreligious, and obsessive, for example, making an excessive number of long distance telephone calls. Her differential diagnoses included: schizoaffective, bipolar, and organic affective disorders. She was then treated with thioridazine and amytriptyline. At age 55, she was noted to have saccadic pursuits, a hypokinetic and ataxic dysarthria, bradykinesia, and her first documented seizure. Between ages 55 and 61, she became demented, rigid, and virtually mute. She was transferred to the Mayo Clinic for evaluation at age 61 .

On neurological examination, the patient was essentially mute. She had craniocervical dystonia, axial rigidity, and stimulus sensitive myoclonus. Eye movement examination was hampered by severe blepharospasm. MRI of the head showed subtle small foci of increased T2 signal changes within pons and cerebral white matter (see fig lA). Bone marrow biopsy revealed a normocellular marrow with increased foamy macrophages. Skin fibroblast testing showed 5.5\% cholesterol esterification and a positive filipin staining consistent with a diagnosis of NPC. ${ }^{3}$ Genetic testing was not performed.

She died at age 62 .

\section{Case 2}

A 32 year old woman presented to Mayo Clinic with a five year history of an undiagnosed neuropsychiatric disorder.

Her birth and development had been unremarkable. At the age of 27, she developed a "nervous breakdown," associated with paranoid delusions and was treated with haloperidol. Later that year, her psychosis resolved, but she developed facial dystonia, a shuffling gait and dysarthria, attributed to a tardive syndrome. Subsequently she experienced an annual recurrence of her psychiatric symptoms, particularly the paranoid delusions. At 32, she became dysarthric, ataxic and

Abbreviations: NPC, Niemann-Pick disease type C; VSGP, vertical supranuclear gaze pulsy 

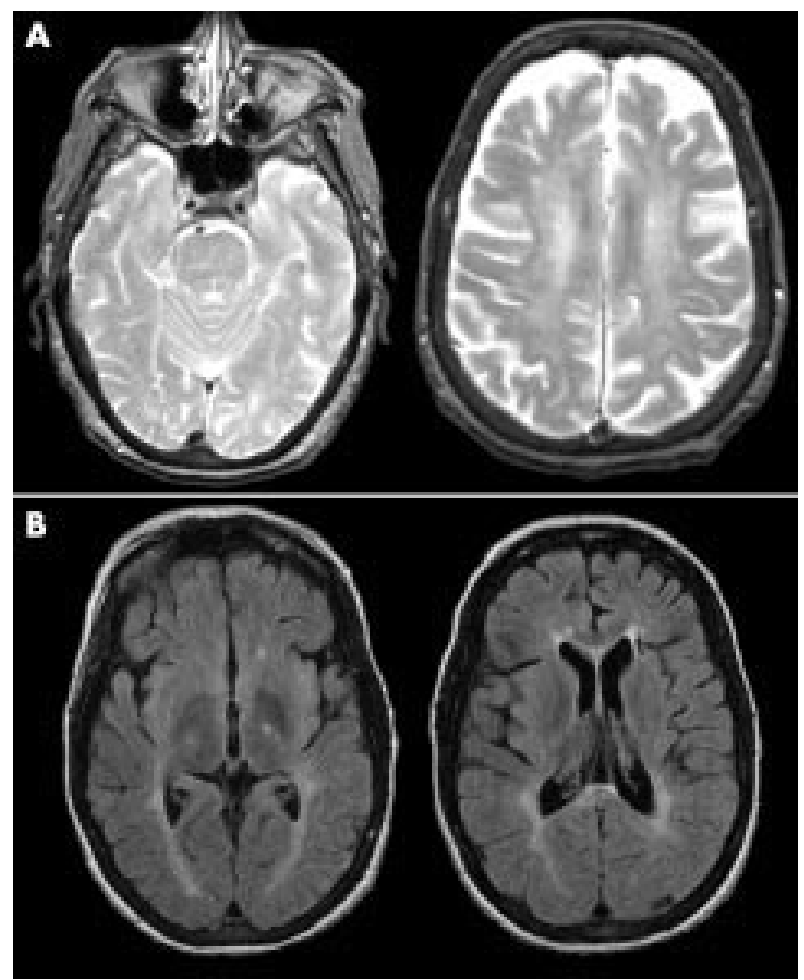

Figure 1 Axial head MRI in adult onset Niemann-Pick disease type C. T2 weighted sequences in case 1 (A) show signal changes within pons and cerebral white matter while fluid attenuated inversion recovery sequences in case 2 (B) show atrophy and increased signal within the periventricular white matter and basal ganglia.

neurological examination revealed a vertical supranuclear gaze palsy (VSGP). She was give intravenous thiamine for possible Wernicke's encephalopathy. A small bowel biopsy was performed revealing lymphangiectasias, "suggestive of Whipple's" and she was treated with intravenous antibiotics. However, her decline continued, and she developed urinary incontinence and insomnia.

Neurological examination at Mayo Clinic revealed oromandibular dystonia, an ataxic and hypokinetic dysarthria, mild hyperreflexia, and gait ataxia. MRI of the head showed increased T2 and fluid attenuated inversion recovery signal in bilateral basal ganglia and periventricular areas (see fig $\mathrm{lB}$ ). Duodenal biopsy was negative. Brain biopsy revealed mild gliosis. One month later, neurological examination revealed virtual mutism, definite corticospinal tract signs, and a VSGP. Bone marrow biopsy demonstrated scattered sea blue histiocytes. Skin fibroblast testing showed 26\% cholesterol esterification and indeterminate filipin staining. She was diagnosed with variant type NPC. ${ }^{3}$ A year later, a repeat cranial MRI showed extensive white matter disease. Genetic testing was not performed.

\section{DISCUSSION}

Of our five confirmed cases with adult onset NPC two (40\%) presented with psychosis. This is higher than the 25\% (4 of 16) noted in the review by Shulman $e^{2} \mathrm{al}^{2}$ and may reflect a referral bias of complicated cases to the Mayo Clinic. Alternatively, the higher prevalence of psychosis at onset noted in this small series of adult onset NPC cases, may, more accurately reflect true prevalence, as our cases were limited to those with cytochemical confirmation.

Patients with inborn errors of metabolism are usually diagnosed in childhood. To date, there are less than 25 reported cases of adult onset NPC. ${ }^{24}$ Compounding the diagnostic confusion surrounding our two patients was their initial symptom: psychosis. In the single extant report of a patient with adult onset NPC presenting with psychosis, several renowned movement disorder authorities underscore that such a case is extraordinary. ${ }^{5}$ Our two patients demonstrate that adult onset NPC presenting with psychosis is not unique, though is probably rare. Our case histories reveal clues that may aid clinicians in recognising similar patients. For example, the presence of a VSGP in a progressive disorder narrows a patient's diagnostic possibilities considerably to: progressive supranuclear palsy, corticobasal degeneration, multiple systems atrophy, dementia with Lewy bodies, Whipple's disease, Wilson's disease, Huntington's disease, neuroacanthocytosis, and NPC. ${ }^{6}$

Another potentially illuminating feature of our two cases is that the patients developed probable tardive parkinsonism and other dyskinesias readily after receiving neuroleptics. This is reminiscent of what occurs in patients with dementia with Lewy bodies who receive neuroleptics. ${ }^{7}$ Additionally, it may be difficult to ascertain whether patients with neuroleptic exposures, who later develop dyskinesias, have a tardive phenomenon or not, ${ }^{89}$ as it was in our patients. In either case, one should consider the possibility of an undiagnosed disease affecting the basal ganglia in psychotic patients who abruptly develop dyskinesias after ingesting neuroleptics.

Organomegaly is common in patients with inborn errors of metabolism, including NPC; however, hepatosplenomegaly may be present in only $50 \%$ of patients with adult onset $\mathrm{NPC}^{2}$ so its absence should not dissuade one necessarily from this diagnosis.

The presence of seizures also is typical in adult onset NPC. ${ }^{2}$ In summary, adult onset NPC may present in adulthood with psychosis. When a patient less than 60 years of age presents with the tetrad of psychosis, a VSGP, movement disorders, and seizures, NPC is a possible aetiology.

\section{Authors' affiliations}

K A Josephs, Department of Neurology, Mayo Clinic, Rochester, USA J A Van Gerpen, Department of Neurology, Ochsner Clinic Foundation, New Orleans, USA

M W Van Gerpen, Department of Psychiatry and Neurology, Tulane University School of Medicine, New Orleans, USA

Competing interests: none declared.

Correspondence to: Dr K A Josephs, Department of Neurology, Mayo Clinic, 200 First Street SW, Rochester, MN 55905, USA; josephs.keith@mayo.edu

Received 19 August 2002

Accepted in revised form 18 November 2002

\section{REFERENCES}

1 Pentchev PG, Vanier MT, Suzuki K, et al. Niemann-Pick disease type c: a cellular cholesterol lipidosis. In: Scriver CR, Beaudet AL, Sly WS, et al, eds. The metabolic and molecular bases of inherited disease. 7th edn. New York, :McGraw-Hill, 1995:2625-39.

2 Shulman LM, David NJ, Weiner WJ. Psychosis as the initial manifestation of adult-onset Niemann-Pick disease type C. Neurology 1995;45: 1739-43.

3 Vanier MT, Rodriguez-Lafrasse, Rousson R, et al. Type C Niemann-Pick disease:spectrum of phenotypic variation in disruption of intracellular LDL derived cholesterol processing. Biochim Biophys Acta 1991;1096:32837

4 Vanier MT, Wenger DA, Comly ME, et al. Niemann-Pick disease group C: clinical variability and diagnosis based on defective cholesterol esterification. Clin Genet 1988;33:331-48.

5 Shulman LM, Lang AE, Jankovic J, et al. What is it? Case 1, 1995: psychosis, dementia, chorea, ataxia and supranuclear gaze dysfunction. Mov Disord 1995;10:257-62.

6 Stell R, Bronstein AM. Eye movement abnormalities in extrapyramida disorders. In: Marsden CD, Fahn S. Movement disorders 3. Oxford: Butterworth-Heinemann, 1994:88-116.

7 Swanberg MM, Cummings JL. Benefit-risk considerations in the treatment of dementia with Lewy bodies. Drug Saf 2002;25:511-23.

8 Jankovic J, Orman J. Tetrabenazine therapy of dystonia, chorea, tics and other dyskinesias. Neurology 1988;38:391-4.

9 Glazer WM, Morgenstern H, Doucette JT. Predicting the long-term risk of tardive dyskinesia in outpatients maintained on neuroleptic medications. J Clin Psychiatry 1993;54:4. 1993. 